\title{
Optical Device for Measuring Small Displacements of Control Objects Surfaces with Protection from External Mechanical Loading
}

\author{
I. P. Miroshnichenko ${ }^{1, *}, I . A$. Parinov $^{2}, T$. P. Savostina ${ }^{1}$, and V. P. Sizov ${ }^{1}$ \\ ${ }^{1}$ Don State Technical University, Rostov-on-Don, Russia \\ ${ }^{2}$ Southern Federal University, Rostov-on-Don, Russia
}

\begin{abstract}
The paper describes high-precision contactless optical devices for measuring small linear and angular displacements of the surfaces of control objects based on modern laser technologies and new methods of optical interferometry. A new scientific-based technical solution has been proposed, which allows one to provide continuous protection against the influence of external mechanical loading in the measurement process. The proposed technical solutions are successfully used to solve actual scientific and industrial problems and can also be successfully used to control the quality of structural materials and diagnostics of the state of goods under operating conditions in mechanical engineering, shipbuilding, aircraft construction, etc.
\end{abstract}

\section{Introduction}

The development and introduction of new high-precision measuring tools and technologies for monitoring the quality of structural materials and diagnostics of the state of goods under operational conditions are currently relevant and require in solving many scientific and applied problems [1-3].

Promising in this case is the use of contactless means of measuring small displacements of the surfaces of control objects based on modern laser technologies and new methods of optical interferometry. This allows one to significantly improve the accuracy and informativeness of the results of the study by acoustic methods of non-destructive testing.

\section{Brief description and analysis of known technical solutions}

The Department of Industrial Electronics and Lighting EIn [4], an optical interference device for measuring small linear displacements was developed, developed on the base of a two-way laser interferometer with a combined branch, described in $[5,6]$, the scheme of which is present in Figure 1.

The device [4] contains optically coupled and sequentially placed source of coherent optical radiation 1 (gas or semiconductor laser), optical system 2, beam splitter 3, reflector 4 fixed on the surface 5 of the test object 6 , and screen 7 , with a photodetector (PD) 8 installed on it. At the same time, the beam splitter 3 and the reflector 4 are located relative to each other under angle $\alpha$, it allows one to obtain the interference pattern
(IP) 9 by combining the reference and objective beams. The IP is a set of rings 10 of various intensities, which are projected onto the screen 7 . The PD 8 is made in the form of a rectangular matrix of photodetectors 11 and is installed in the plane of the screen 7 radially to the rings 10 of IR 9. It locates at a distance from the edge 12 of the IR 9 opposite to the apex of angle $\alpha$ between the beam splitter 3 and the reflector 4 , to center IR 9. The PD 8 is connected to a device for recording, processing and displaying results.

The principle of the device operation [4] is as follows. The radiation from source 1 after passing through the optical system 2 is converted into a diverging beam and falls on the beam splitter 3 where it separates. First part reflects from the surface of beam splitter 3 (reference beam), and the second part reflects from reflector 4 (object beam), fixed on the surface 5 of the control object 6 . Then IP 9, obtained by combining the reference and object beams projects onto the screen 7 with the PD 8 installed on it.

It may be possible that the reflector 4 is absent, and the reflecting surface 5 of the control object 6 itself directly performs its functions.

The change in the intensity of the IP rings from the minimum to the maximum value occurs when the surface of the control object moves by the value of $\lambda / 4$, where $\lambda$ is the optical radiation wavelength (for example, for optical radiation of a helium-neon gas laser, red, $\lambda=$ $0.63 \mu \mathrm{m})$.

The PD 8 with a linear displacement of the surface 5 of the control object 6 measures the intensity of the optical field in the region of IP, which has the maximum information and other characteristics (high contrast, dynamic range, etc.). The results of measurements of the

\footnotetext{
* Corresponding author: ipmir2011@yandex.ru
} 
intensity of the optical field of IP, uniquely associated with the linear displacement of the surface 5 of the control object 6 , are then transmitted from the PD 8 for recording, processing and displaying the measurement results.

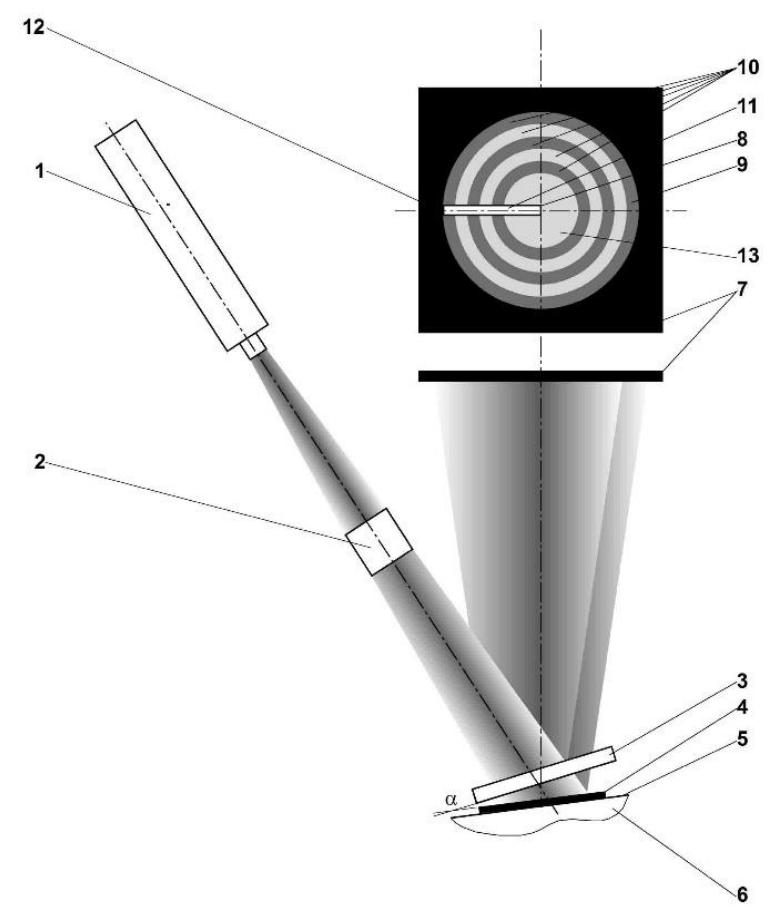

Fig. 1. Scheme of the device for measuring small displacements.

Local overpotentials in high-voltage insulators, Possible options for recording the intensity in this case can be the registration of the total intensity of the optical field on the surface of the PD 8, the registration of the intensity of the optical field in a ring of the same order, in the rings of the first and second orders, etc. So. device [4] provides the implementation of almost all known options for recording information from IP.

The device [4] is scientifically substantiated, its technical and functional characteristics have been adequately determined and studied during theoretical and experimental research carried out using prototypes, the results of which are given in [7-12].

To expand the functionality of devices [4-6] due to the simultaneous measurement of small linear and angular displacements of the surfaces of control objects, which is most important, when solving practical problems, where only linear displacements are quite rare, we have proposed [13-15] a new scientifically based method, a method and a device [16]. The results of their design and experimental studies were published in [13$15,17-20]$.

Monitoring the operation of devices [4-6,16] in the process of measuring and eliminating the influence of "internal" destabilizing factors on their results (voltage fluctuations in the power network, changes in the radiation intensity of the source, microdefects of optical circuit elements, etc.), reducing the quality of measurement results can be achieved by using the new approach [21,22] and the method [23] of a complex correction of the measurement results of small displacements by optical interference means. These methods consist in that the total intensity of the optical field over the IP area is registered simultaneously and continuously during measurements. By using the magnitude of IP area, it is determined the correction introduced "in the same time scale" in the result of measurements.

\section{Description of the proposed technical solution}

At present, a significant drawback inhibiting the use of known high-precision optical devices for measuring displacements, based on the use of interference methods, including devices [4-6, 16], is the low quality of measurement results. It is caused by the influence on them in the process of measuring, the external unsteady and, as a rule, random mechanical effects (vibrations of technological test equipment, mechanical effects of various nature, etc.). They cause additional displacements and introduce random errors in the measurement results. Manifestations of this deficiency are especially evident by using optical interference measuring instruments as part of mobile diagnostic systems, i.e. when measurements take place in "field" conditions.

A new technical solution has been developed to ensure the continuous protection of known devices [4$6,16]$ against the influence of external mechanical influences in the process of measuring by registering and simultaneously compensating for small movements of the surfaces of control objects caused by external mechanical effects and affecting the measurement results. It has been protected by patent of the Russian Federation for invention [24].

The scheme of the proposed device is present in Figure 2, and Figure 3 shows an enlarged image of the screen with the PD located in its plane.

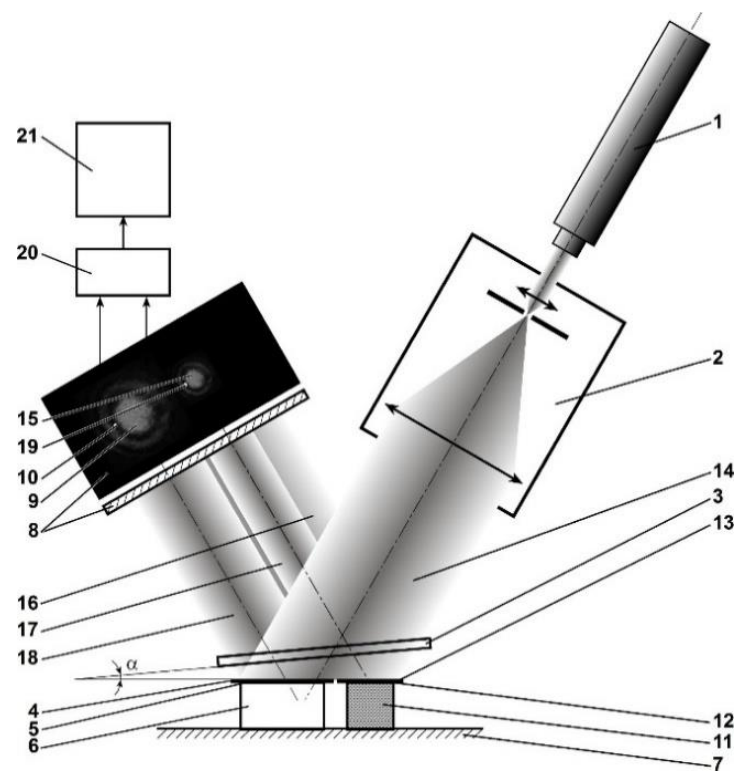

Fig. 2. Scheme of device. 


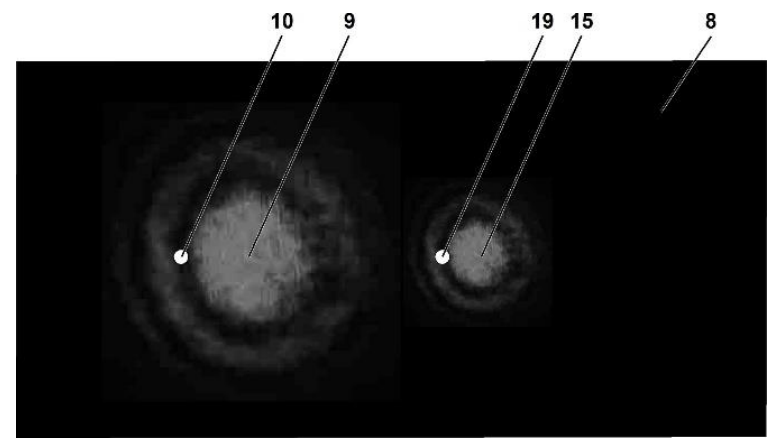

Fig. 3. External imagine of screen with PD.

The device [24] contains optically coupled and sequentially placed a source of coherent optical radiation 1; an optical system 2; a beam splitter 3; a reflector 4, mounted on the surface 5 of the control object 6 , rigidly attached to the support base 7, and located under angle $\alpha$ to the beam splitter 3; the screen 8 with PD 10, installed in screen plane, in a given area of IP 9.

The device also includes a simulator 11 of the control object with an additional reflector 13 installed on its surface 12 . The simulator 11 is rigidly fixed on the support base 7 in the area irradiated by the optical radiation beam 14 behind the optical system 2 . It is provided with devices for adjusting the position of the additional reflector 13 . The additional reflector 13 is placed in one plane with a reflector 4 fixed on the surface 5 of the control object 6 . On the screen 8, IP 15 is additionally projected, which is obtained by combining the reference beam 16 from the beam splitter 3 and the object beam 17 from the additional reflector 13. It is stated an additional PD 19 installed in the plane of the screen 8 in its area, similar to the IP 9, obtained by combining the reference beam 16 from the beam splitter 3 and the object beam 18 from the reflector 4 , mounted on the surface 5 of the test object 6 . So PD 10 and additional PD 19 are installed, respectively, in the rings of the first order IP 9 and 15 (see Figure 3). The outputs of the PDs 10 and 19 are connected to the inputs of the differential amplifier 20, the output of which is connected with the device 21 of recording, processing and displaying the measurement results.

The principle of the device operation is as follows. Before starting testing, the control object 6 is rigidly fixed on the base 7 with the reflector 4 , installed on its surface 5 and the control object simulator 11 with an additional reflector 13 , installed on its surface 12. After this, the optical measuring circuit is assembled by sequential arrangement and providing optical communication of the source 1 , the optical system 2 , the beam splitter 3 .

At the same time, the reflector 4 and the additional reflector 13 are located under angle $\alpha$ to the beam splitter 3 . The test object 6 with the reflector 4 and the simulator 11 with the additional reflector 13 are located on the support base 7 in the area irradiated by the optical radiation beam 14 behind the optical system 2 . Next, the position of the additional reflector 13 is adjusted using the test object simulator 11 configured to perform this operation by placing it in the same plane with the reflector 4 in such a way that IPs 9 and 15 are projected on the screen 8 . These IPs are obtained respectively by combining the reference beam 16 from the beam splitter 3 and the object beam 18 from the reflector 4 and the reference beam 16 from the beam splitter 3 and the object beam 17 from the additional reflector 13. In the plane of the screen 8 in the similar predetermined regions, IPs 9 and 15, the PD 10 and the additional PD 19 are installed. Their outputs are electrically connected to the differential amplifier 20. The output of the differential amplifier 20 is connected to the device 21 of the registration, processing and display of measurement results. In the process of testing, the PD 10 and 19 register the intensity of the optical field at their installation sites in specified areas of IPs 9 and 15 .

The signals of the PD 10 and 19 are transmitted to the inputs of the differential amplifier 20. Here the signal of the additional PD 19, containing information about the displacements of the reflector 13 , caused by random disturbances, is subtracted from the signal of the PD 10 . The PD 10 contains information about the displacements of the reflector 4 , caused by the total displacements of the surface 5 of the test object 6 ("useful signal") in the control process and random disturbances. Then, the signal from the output of the differential amplifier 20, containing only information about the displacements of the surface 5 of the test object 6 in the process of control, is transmitted to the input of the device 21 for recording, processing and displaying the measurement results. In the device 21, the correspondence of the measured intensity is determined, which is uniquely associated with the displacement of the surface 5 of the control object 6 , its registration and displayment of the obtained measurement result.

To confirm the principal feasibility of the implementation of the proposed device and conduct an experimental study of its technical and functional characteristics, an experimental setup was developed. The scheme of this setup was similar to that shown in Figure 2.

During the tests, the following options were modeled:

(i) with a fixed (constant) displacement of the surface 5 of the test object 6 , harmonic oscillations were applied to the supporting base 7 , imitating an external mechanical loading;

(ii) when a pulsed displacement of the surface 5 of the test object 6 , harmonic oscillations were applied to the supporting base 7 , imitating an external mechanical loading.

In the process of testing, signals were recorded at the outputs of the PD 10, the additional PD 19 and the differential amplifier 20, at the same time, the PD 10 and 19 were installed in the screen plane in the first-order rings IPs 9 and 15, respectively. The test results are present in the form of oscillograms in Figure 4, respectively, for the first (a) and second (b) of the noted modeling options. In Figure 4, the curves 1 present the signals at the output of the PD 10, the curves 2 show signals at the output of the PD 19 and the curves 3 demonstrate the signals at the output of the amplifier 20. 


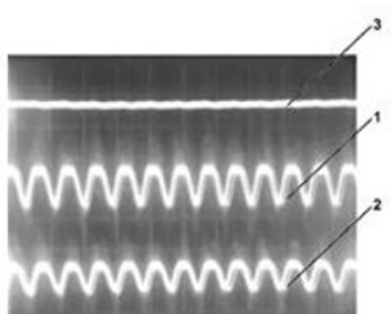

a.

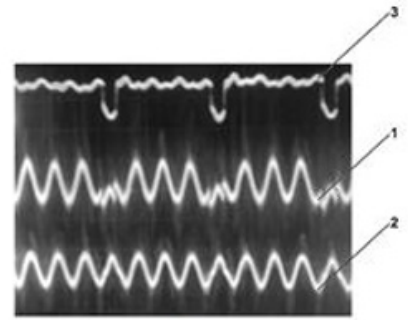

b.
Fig. 4. Test results.

Analysis of the results allows us to conclude about the feasibility of the proposed method and the solution of the task of development, namely, improving the quality of the measurement results of small displacements of the surfaces of the test objects.

\section{Conclusions}

The paper describes high-precision contactless means of measuring small linear and angular displacements of surfaces of test objects based on modern laser technologies and new methods of optical interferometry, and also proposes a new scientifically-based technical solution to ensure their continuous protection from the effects of external mechanical influences during measurements.

The proposed technical solutions are successfully used to solve actual scientific and industrial problems and can also be successfully used to control the quality of structural materials and diagnose the state of goods under operating conditions in mechanical engineering, shipbuilding, aircraft construction, etc.

The work was supported by the grant of Russian Foundation for Basic Research, No. 19-08-00365, Ministry of Education and Science of the Russian Federation: projects Nos. 3.5378.2017/VU (organization of the implementation of researches), 9.9770.2017/BCh (the basic part of the state task).

\section{References}

[1] M.Yu. Fedotov, O.N. Budadin, S.O. Kozelskaya, Mathematical modeling and experimental results of control of PCM by fiber-optic sensors taking into account the influence of factors simulating real operating conditions Testing. Diagnostics 4, 12-19 (2019)

[2] O.N. Budadin, E.S. Vyachkin, E.A. Vyachkina, V.O. Kaledin, S.O. Kozelskaya, Experimental investigations of ultrasonic thermal tomography (us thermotomography) of composite materials Testing. Diagnostics 6, 58-62 (2019)

[3] M.Yu. Fedotov, O.N. Budadin, S.O. Kozelskaya Technological aspects of creating a fiberoptic non-destructive testing of sandwich composite structures Testing. Diagnostics 7, 24-29 (2019)

[4] I.P. Miroshnichenko, I.A. Parinov, E.V. Rozhkov, A.G. Serkin, Optical device for measuring displacements Patent RU 2373492, (In Russian) (2009)
[5] A.N. Matveev, Optics. Moscow: High School.- 351 (In Russian) (1985)

[6] A.S. Batrakov, M.M. Butusov, G.P. Grechka, Laser Measurement systems: Moscow: Radio and Communication. 456 (In Russian) (1981)

[7] I.P. Miroshnichenko, A.G. Serkin, The results of experimental studies of the interference meter of small displacements Measuring Equipment 5, 22-25 (2006)

[8] I.P. Miroshnichenko, A.G.V.P. Serkin, Features of the use of interference meters of small displacements when solving problems of diagnosing the technical condition of constructions Russian Journal of Non-destructive Control 4, 31-38 (2007)

[9] I.P. Miroshnichenko, A.G.V.P. Serkin, Experimental justification of proposals for improving the interference meters for small displacements Proceedings of the Rostov State Transport University 1(8), 85-90 (In Russian) (2009)

[10] I.P. Miroshnichenko, A.G. Serkin, V.P. Sizov, Electrodynamic analysis of laser interferometers General Issues of Radio Electronics. Scientific and technical collection 155-161 (In Russian) (2010)

[11] I.P. Miroshnichenko, Perspective optical means of measuring small displacements for systems of diagnosing the technical state of materials and goods Control. Diagnostics 1, 45-49 (2010)

[12] V.E. Alekhin, I.P. Miroshnichenko, A.G. Serkin, Laser interferometer for measuring displacements Nanoindustry 2, 38-41 (In Russian) (2010)

[13] I.P. Miroshnichenko, A.G. Serkin, V.P. Sizov, Numerical study of the measurement method of small linear and angular displacements by laser interferometers Measuring Equipment 1, 9-13 (2007)

[14] I.P. Miroshnichenko, A.G. Serkin, V.P. Sizov, Unified method for measuring small spatial displacements by laser interferometers News of Universities.Instrument Making 12, 34-40 (2007)

[15] I.P. Miroshnichenko, A.G. Serkin, Unified method for measuring small linear and angular displacements of control objects surfaces Russian Journal of Non-destructive Control 5, 23-27 (2008)

[16] I.P. Miroshnichenko, A.G. Serkin, V.P. Sizov, Measuring method of linear and angular displacements Patent RU 2388994, 10.05.2010 (In Russian)

[17] I.P. Miroshnichenko, A.G. Serkin, V.P. Sizov, Using a point source of radiation to extend the functionality of a displacement meter Optical Journal 7, 41-47 (2008)

[18] I.P. Miroshnichenko, A.G. Serkin, V.N. Prokopez, On the use of diffraction gratings in promising laser interferometers Proceedings of the Rostov State Transport University 1(8), 107-113 (In Russian) (2009)

[19] I.P. Miroshnichenko, A.G. Serkin, On the use of optical interference methods for measuring linear and angular displacements in the diagnostic systems of the technical state of materials and goods Control.Diagnostics 7, 46-50 (2011)

[20] I.P. Miroshnichenko, Parinov I.A., E.V. Rozhkov, A.G. Serkin, V.P. Sizov, Novel Test Means and Techniques Based on Optical Interferometry and 
Acoustic Emission to Study Displacements of Object Surfaces and Damage of High-Temperature Superconductive Tapes and Related Composites Piezoelectrics and Related Materials: Investigations and Applications, Ivan A.Parinov (Ed.) (New York: Nova Science Publishers) 239-282 (2012)

[21] I.P. Miroshnichenko, A.G. Serkin, Correction of measurement results of small displacements by laser interferometers Sensors and Systems 3, 28-30 (2008)

[22] I.P. Miroshnichenko, A.G. Serkin, Complex correction in laser diagnostics of displacements Photonics 5(17), 18-20 (2009)

[23] Alekhin V.E., I.P. Miroshnichenko, A.G. Serkin, Sizov V.P., The method of recording displacements by optical sensors Patent RU 2343403, 10.01.2009 (In Russian)

[24] I.P. Miroshnichenko, V.A. Nesterov, A.G. Serkin, V.P. Sizov, V.A. Shevtsov, Interference meter of small displacements Patent RU 2410642, 27.01.2011 (In Russian) 